\title{
I | 4 I Can every other heart-beat acquisition be better for the same breath-hold interval in MR tagging sequences?
}

\author{
Tamer Basha*, El-Sayed Ibrahim, Monda L Shehata, Robert G Weiss and \\ Nael F Basha
}

\begin{abstract}
Address: Johns Hopkins University, Baltimore, MD, USA
\end{abstract}
* Corresponding author

from I th $^{\text {th }}$ Annual SCMR Scientific Sessions

Los Angeles, CA, USA. I-3 February 2008

Published: 22 October 2008

Journal of Cardiovascular Magnetic Resonance 2008, I0(Suppl I):A266 doi:I0.I I86/I532-429X-I0-SI-A266

This abstract is available from: http://jcmr-online.com/content//0/SI/A266

(c) 2008 Basha et al; licensee BioMed Central Ltd.

\section{Introduction}

In cardiac MRI with segmented k-space acquisition, data is partially acquired through consecutive cardiac cycles. However, the available longitudinal magnetization in a given cardiac cycle is exhausted by the excitation radiofrequency pulses in previous cycles, which limits the available signal-to-noise ratio (SNR). One potential solution is to increase the repetition time (TR) to allow for partial magnetization recovery between the RF pulses. However, this solution is not practical in sequences with breathhold acquisition as it increases the breath-hold time, which limits the applicability of the considered sequence. In this work, a new technique is proposed for improving the SNR without increasing the breath-hold time. The idea is based on acquiring the signal every other heartbeat within the same breath-hold time interval. This limits the number of the acquired k-space segments to a half but allow the magnetization a longer time to recover. This technique results in better SNR, compared to conventional (every heartbeat) segmented k-space acquisition, as evidenced by numerical simulations of the Bloch equation as well as phantom and in-vivo experiments. Two tagging pulse sequences are considered in this work: complementary-spatial-modulation-of-magnetization (CSPAMM) [1] and strain-encoding (SENC) MRI [2].

\section{Methods}

Numerical simulation

Numerical simulations of the Bloch equation were conducted to examine the effect of the imaging flip angle and
TR on the resulting signal amplitude. Ramped flip angle scheme was implemented $[1,3]$. The simulations were conducted for both every heartbeat and every-other heartbeat acquisition schemes.

\section{Phantom experiment}

To verify the simulations, a phantom experiment was conducted using a spiral acquisition [4]. Two sets of CSPAMM and SENC images were acquired using the same breathhold intervals. In the first set, the acquisition was done every heartbeat while for the second set it was every-other heartbeat. To compensate for the number of spiral trajectories that was halved in the second set, two modifications were done. First, the spiral acquisition window time was doubled. Second, a multi-shot acquisition with factor 2 per cardiac phase was used.

\section{In vivo studies}

Two normal volunteers were consented and scanned using the same sequences. SNR was measured from the images by defining a region of interest in the static tissue and the heart. The noise was measured from the background and adjusted for Rayleigh distribution.

\section{Results and discussion}

Simulation results are shown in Figs. 1 and 2. Fig. 3 shows the SNR gain of the every other heartbeat acquisition over every heartbeat acquisition. Fig. 3.a shows the gain in the phantom study using the two mentioned maneuvers. Fig. 3.b shows the SNR gain in-vivo using the first maneuver 


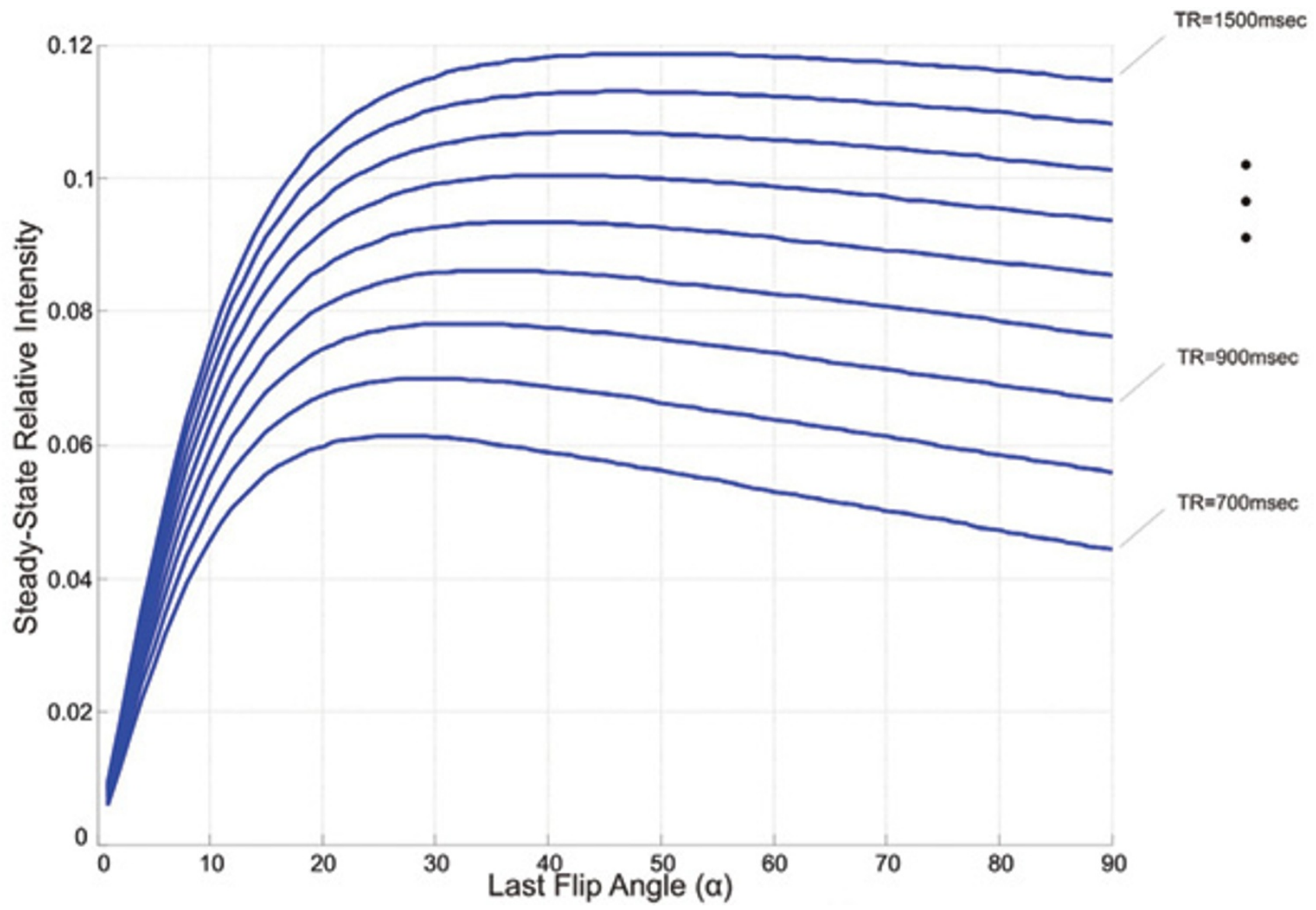

Figure I

Relative intensity of the tagging steady-state vector for different last flip angle and different repetition time (TR).

only. The results show that the every-other heartbeat acquisition performs better than every heartbeat acquisition. However, the first maneuver has a substantial increase in SNR $(\approx 180 \%)$ at the nominal flip angle values $\left(<40^{\circ}\right.$ due to the SAR limitations during actual scans). Finally, Fig. 4 shows short-axis cardiac images acquired with every- and every-other heartbeat for different flip angles. We can easily distinguish the SNR enhancement in both the myocardium and the background noise.

\section{Conclusion}

In contrast to what is usually performed in routinely conducted CSPAMM and SENC studies, we have demonstrated that for the same breath-hold time interval, the every-other heartbeat acquisition gives substantially higher SNR than every heart-beat acquisition. This should be taken into consideration for routinely conducted studies to improve the quality of the resulting images.

\section{Acknowledgements}

This work was supported in part by grants from the national institute of heart, lung and blood R0IHL072704 and ROIHL61912, and Donald W.

Reynolds Foundation grant.

\section{References}

I. Fischer, et al.: MRM 30:191-200.

2. Osman, et al.: MRM 46:324-334.

3. Stuber, et al.: MAGMA 9:85-91.

4. Ryf, et al:: MRM 5 I:237-242. 


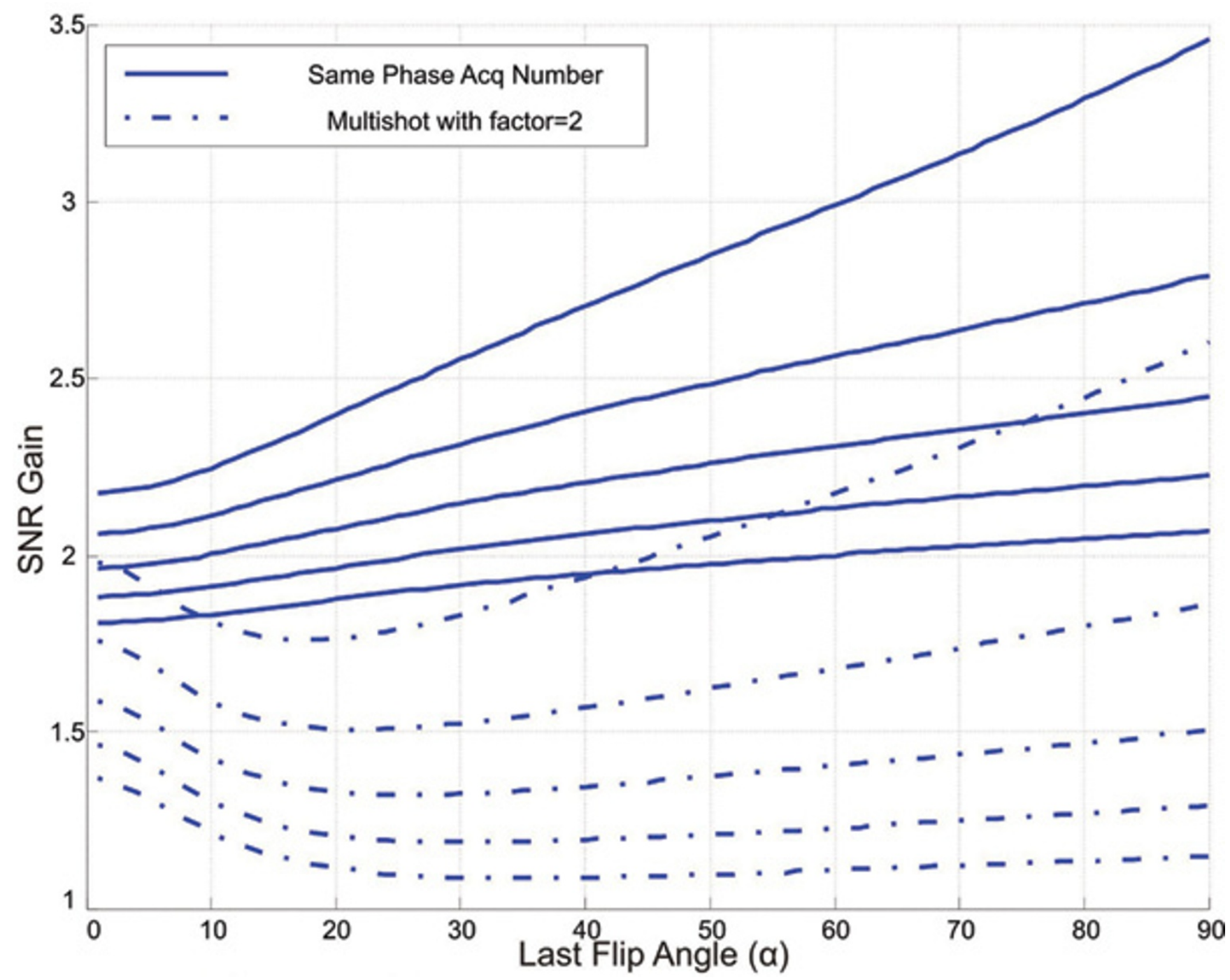

\section{Figure 2}

Gain in the SNR of every other heart-beat acquisition over every heart-beat acquisition for different last flip angle and different repetition time (TR). The solid lines represent the gain using the maneuver of doubling the acquisision time while the dotted line shows the gain for the maneuver of using multishot acquisition with factor $=2$. 


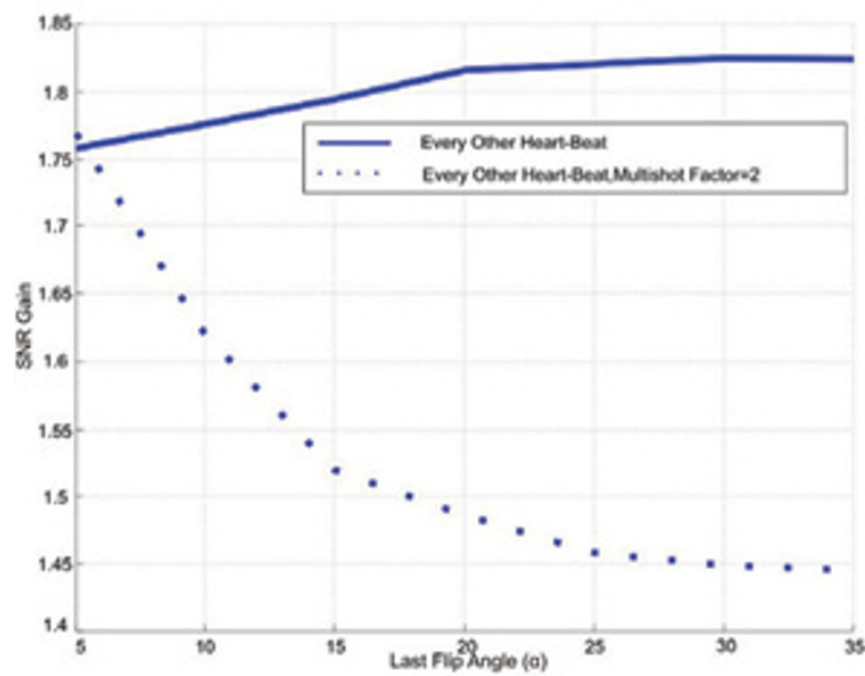

(a)

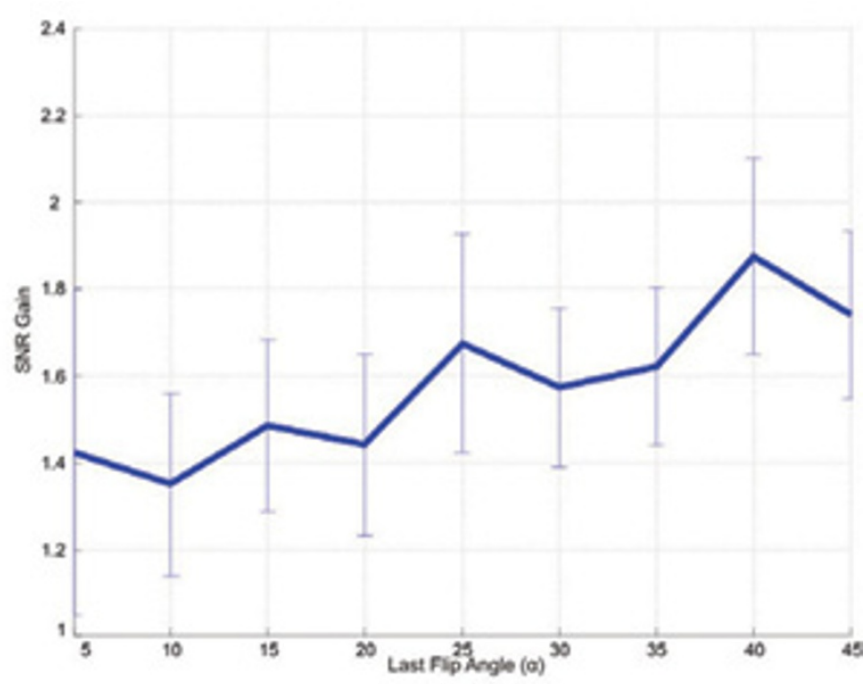

(b)

Figure 3

SNR gains of every other heart-beat acquisition over every heart-beat acquisition for different last flip angle. 20 cardiac phase images were acquired with temporal resolution of $20 \mathrm{~ms}$ and one breath-hold scar time of $17 \mathrm{sec}$. The every heartbeart acquisition consists of 16 spiral with spiral window of $8 \mathrm{~ms}$. (a) In phantom, using the two maneuvers. The solid line represents the gain using the maneuver of doubling the acquisition time (the acquisition consist of 8 spirals with spiral window of 16 ms) while the dotted line shows the gain for the maneuver of using multishot acquisition with factor $=2$ ( 16 spirals with spiral window of $8 \mathrm{~ms}$ ). (b) In vivo, the bars represent =/- two standard deviation from the mean value of the SNR gain. 
(a)
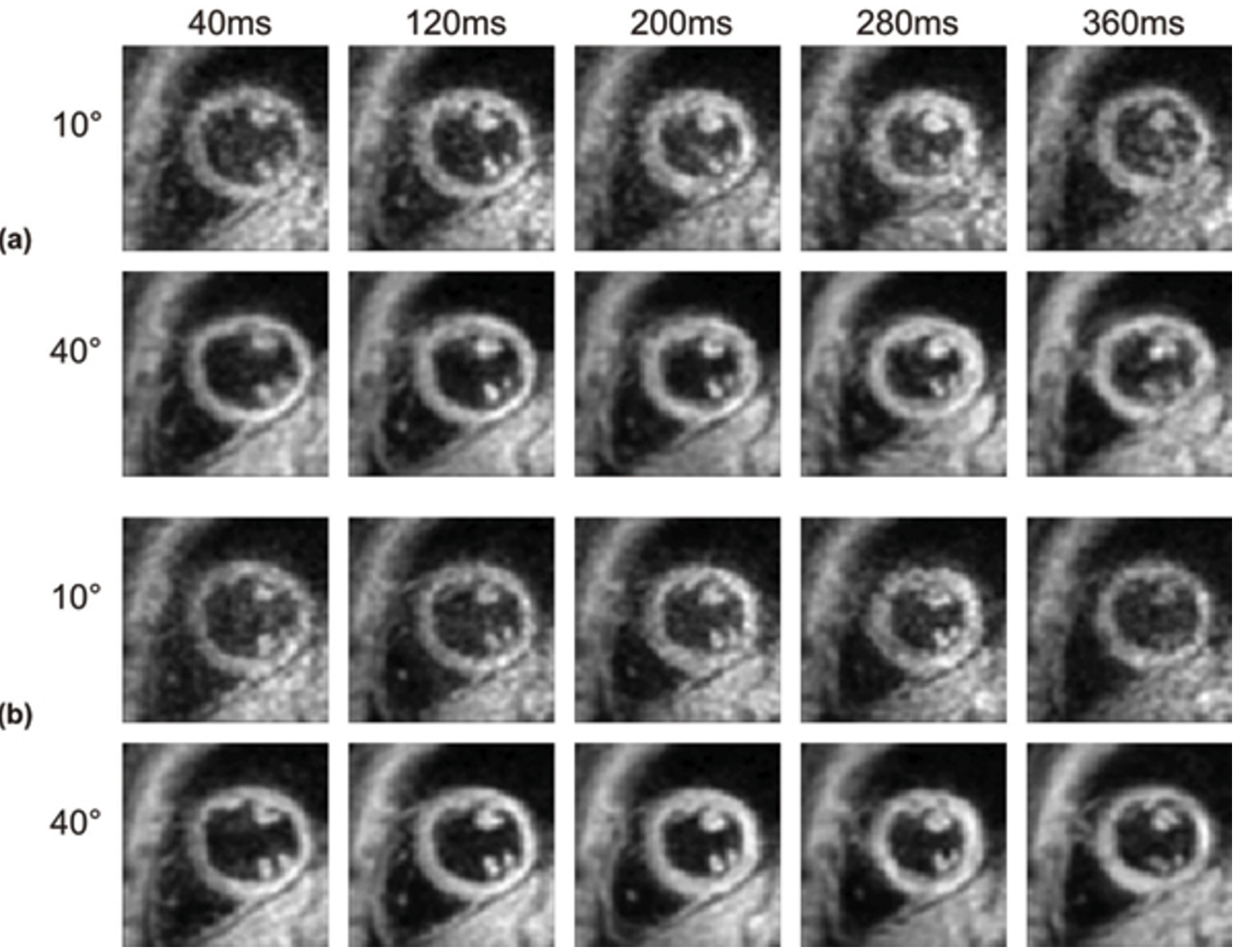

Figure 4

SENC Short-axis images for cardiac phases during early systole at location corresponding to the left ventricular basal section and at two different flip angles using: (a) Every Heart-Beat, and (b) Every Other Heart-Beat. For each image, the acquisition time, measured directly after the R-wave, appears above its column, and the used flip angle appears to the left of its row. The imaging parameters are 20 cardiac phases with temporal resolution of $20 \mathrm{~ms}$. For the every heartbeat, 16 spiral was acquired with spiral window of $8 \mathrm{~ms}$. For the every-other heartbeart, 8 spiral was acquired with spiral window of $16 \mathrm{~ms}$.

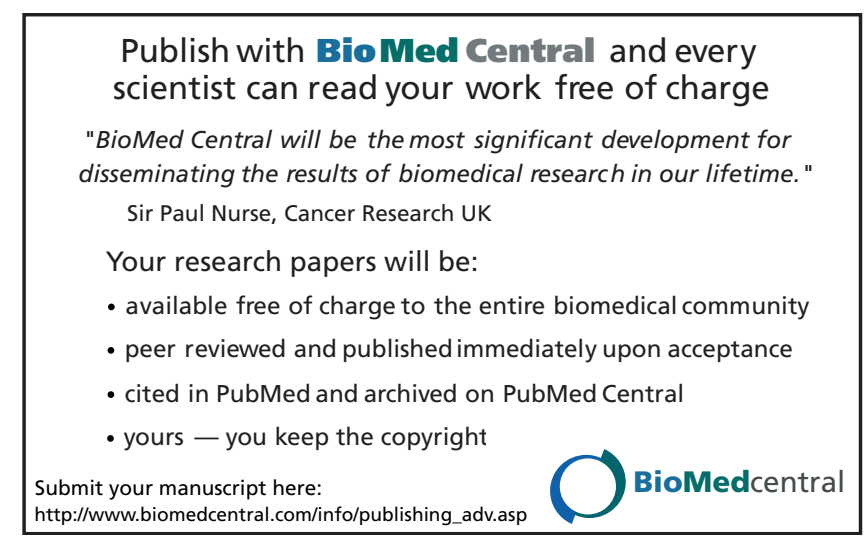

